\title{
Pasteurella multocida Endocarditis with Septic Arthritis: Case Report and Review of the Literature
}

\author{
Authors: \\ ${ }^{\dagger}$ E Carter, ${ }^{1++}$ U Iroegbu, ${ }^{1}$ W Baig, ${ }^{1}$ JAT Sandoe 1,2 \\ 1. Leeds Teaching Hospital Trust, UK \\ 2. University of Leeds, UK \\ ${ }^{*}$ Correspondence to uchechika.iroegbu@nhs.net \\ +These authors contributed equally to the work. \\ Disclosure: The authors have declared no conflicts of interest. \\ Disclaimer: The authors have declared no conflicts of interest. \\ Received: $\quad 12.06 .21$ \\ Accepted: $\quad 10.08 .21$ \\ Keywords: Ciprofloxacin, Pasteurella multocida, penicillin, prosthetic-valve infective endocarditis, \\ septic arthritis. \\ Citation: $\quad$ EMJ Microbiol Infect Dis. 2021; DOI/10.33590/emj/21-00129.
}

\section{Abstract}

Background: There is a paucity of evidence regarding optimal management of Pasteurella spp. endocarditis. The authors report the first case of Pasteurella spp. endocarditis with septic arthritis and review the literature.

Case Description: A 79-year-old patient with significant comorbidities, including prosthetic aortic valve, was admitted with left knee swelling, fever, and confusion, having been scratched by a cat 2-weeks prior. At presentation, there was a metallic click, a Grade 3 pan-systolic murmur and Grade 1 flow murmur audible on auscultation. Blood and synovial fluid cultures both isolated Pasteurella multocida, identified by matrix-assisted laser desorption ionisation-time of flight, which was sensitive to penicillin according to the European Committee on Antimicrobial Susceptibility Testing (EUCAST); minimum inhibitory concentration: 0.094). The patient underwent joint washout and received intravenous piperacillin/tazobactam for 3 days before switching to benzylpenicillin once sensitivities were known. Due to continued pyrexia, a transthoracic echocardiogram was obtained, which revealed a small mobile mass on a thickened mitral valve suspicious for a vegetation. On review by the Infective Endocarditis team, conservative management was deemed best, given the presence of comorbidities. Despite requiring further joint washout due to persistent knee pain, the patient was successfully treated with 8 weeks of antibiotic therapy (24 days of benzylpenicillin monotherapy, 2 weeks of benzylpenicillin and ciprofloxacin, and 15 days ciprofloxacin monotherapy).

Discussion: Previous literature reviews report a higher mortality of Pasteurella spp. endocarditis when managed without cardiac surgery, thus recommending surgery in all cases. The authors found these to have confounding factors, including inadequate duration of antimicrobials, aortic root abscess, and rapid progression to death. The authors' case of Pasteurella spp. endocarditis, complicated by septic arthritis, showed successful therapy without cardiac surgery. 


\section{BACKGROUND}

Pasteurella multocida (formerly Pasteurella septica) is a gram-negative aerobic and facultatively anaerobic coccobacillus. It is non-motile, non-sporing, capsulated, and acts as a commensal or opportunistic pathogen in a variety of animal species. Animal to human transmission occurs occasionally, following a break in tissue leading to transmission of the bacteria from the animal to the human. This can be in the form of bites or scratches. Human infections commonly present as a localised abscess or cellulitis and in severe cases, osteomyelitis. Rare manifestations include meningitis, pericarditis, blood stream infection or infective endocarditis.'

P. multocida infections usually respond to penicillin, with tetracyclines, macrolides, and cotrimoxazole as possible alternatives with patients who are allergic to penicillin. Treatment of invasive infections such as infective endocarditis is challenging because of the rarity of the condition and a lack of evidence to inform treatment, especially in patients where a surgical approach is not suitable.

In this report, the authors describe a case of prosthetic valve $P$. multocida infective endocarditis, which was treated conservatively.

\section{CASE PRESENTATION}

A 79-year-old patient presented to hospital with history of left knee swelling, pain, increased confusion, poor appetite, and pyrexia. Collateral history obtained from their spouse revealed a cat scratch 2 weeks prior to presentation. Past medical history included metallic aortic valve replacement, tissue mitral valve replacement, a pacemaker, Type 2 diabetes, Alzheimer's dementia, anxiety, breast cancer, and endometrial cancer with hysterectomy (2001). At initial presentation, clinical examination showed an alert and mildly confused patient with a temperature of $37.9^{\circ} \mathrm{C}$. There were no peripheral stigmata of infective endocarditis; however, a metallic click, consistent with the replaced aortic valve, a Grade 3 mitral pan-systolic murmur, and Grade 1 aortic flow murmurs were both audible on auscultation. There were no signs of pulmonary oedema or aortic regurgitation. The left knee was swollen, warm to touch with limited flexion and extension of the joint, and a working diagnosis of septic arthritis was made.

Blood cultures were taken, and a gram-negative bacillus was identified on the initial blood culture gram stain. Input from the microbiology team advised that the patient be switched from empirical intravenous (IV) flucloxacillin to IV piperacillin-tazobactam to provide adequate gram-negative cover. On matrix-assisted laser desorption ionisation-time of flight (MALDI-TOF) testing, $P$. multocida was identified from the blood cultures. The patient was taken to theatre for aspiration and washout of the knee joint. The knee joint aspiration produced purulent synovial fluid and $P$. multocida was cultured from synovial and tissue samples sent from the knee (identified using MALDI-TOF). The isolate was found to be susceptible to penicillin on to the European Committee on Antimicrobial Susceptibility Testing (EUCAST) disc sensitivities and subsequent penicillin minimum inhibitory concentration of $0.094 \mathrm{mg} / \mathrm{L}$; therefore, the patient was switched to IV of benzylpenicillin of $1.2 \mathrm{~g}$ every 6 hours.

The patient remained pyrexial, despite being on the appropriate antibiotics and joint washout, prompting the arrangement of a transthoracic echocardiogram to look for other sources of deep-seated infection. This showed a small mobile mass on the mitral valve, consistent with a vegetation (Figure 1). The non-microbiology investigations for this case and timeline to diagnosis of suspected endocarditis are summarised in Table 1.

The case was reviewed by the infective endocarditis team and discussed thereafter at the cardiac multidisciplinary team meeting. Due to significant comorbidities, transoesophageal echocardiography and cardiac surgery were deemed not to be suitable in this case. It was agreed that management should be conservative with transthoracic echocardiograms for imaging follow-up.

The patient was treated with benzylpenicillin monotherapy for 24 days, but dosing frequency was increased from $1.2 \mathrm{~g}$ every 6 hours to $1.2 \mathrm{~g}$ every 4 hours on Day 30 because of persisting pyrexia. Oral ciprofloxacin, 500 mg every 


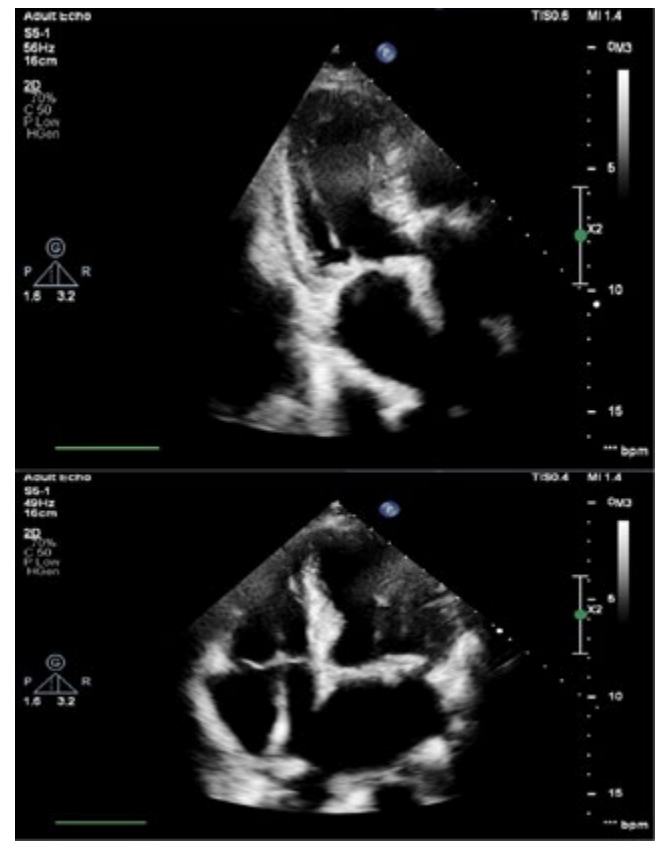

Figure 1: Echocardiogram images showing mitral valve with small mobile mass suspicious of vegetation.

Table 1: Non-microbiology investigations in a patient presenting with Pasteurella multocida septic arthritis and infective endocarditis.

\begin{tabular}{|l|l|l|}
\hline $\begin{array}{l}\text { Days from time of } \\
\text { clinical presentation }\end{array}$ & Investigation & Results and findings \\
\hline 1 & Knee X-ray & $\begin{array}{l}\text { Moderate volume joint effusion, several medial tibiofemoral and } \\
\text { patellofemoral compartment osteoarthritis with bony remodelling, } \\
\text { sclerosis, and osteophytosis } \\
\text { No acute fracture }\end{array}$ \\
\hline 2 & $\begin{array}{l}\text { Arthroscopic } \\
\text { washout of knee }\end{array}$ & $\begin{array}{l}\text { Copious purulent fluid from the joint } \\
\text { Washed out and sent to microbiology }\end{array}$ \\
\hline 3 & TTE & $\begin{array}{l}\text { Metallic AVR in situ } \\
\text { Tissue MVR in situ } \\
\end{array}$ \\
& $\begin{array}{l}\text { Small, mobile mass on MV, consistent with vegetation } \\
\text { Preserved LV systolic function } \\
\end{array}$ & $\begin{array}{l}\text { Moderate RV systolic impairment. } \\
\text { Severe TR }\end{array}$ \\
\hline
\end{tabular}

AVR: aortic valve replacement; LV: left ventricle; MV: mitral valve; MVR: mitral valve replacement; RV: right ventricle; TR: tricuspid regurgitation; TTE: transthoracic echocardiography. 
12 hours, was then added as a second agent based on static raised $\mathrm{C}$-reactive protein and ongoing knee pain ( $P$. multocida isolate was susceptible to ciprofloxacin). The patient had a further joint washout 35 days after commencement of IV benzylpenicillin. On Day 40, IV benzylpenicillin was stopped, ciprofloxacin monotherapy continued, and the indwelling vascular long line was replaced with a peripheral cannula. Repeat blood cultures and synovial fluid cultures from this point onwards were negative. The patient continued to make a good recovery following this and was discharged to a nursing home following a 60-day inpatient stay, without need for further orthopaedic or cardiology follow-up. The total duration of effective antibiotics was 56 days ( 8 weeks). This included 3 days on IV piperacillintazobactam, 24 days of IV benzylpenicillin monotherapy, 2 weeks on dual agent therapy of benzylpenicillin and oral ciprofloxacin and 15 days on ciprofloxacin monotherapy.

\section{LITERATURE REVIEW}

\section{Method}

A local database of all infective endocarditis cases at Leeds Teaching Hospital Trust, UK, was searched for cases involving Pasteurella spp. and this case was the only one identified since 1998. A literature search was conducted via PubMed using the search terms 'Pasteurella' AND 'endocarditis'. Further references were then identified from the references used in these papers. Only case reports written in English, French, or Spanish were included in the analysis, due to the availability of interpretation services. The modified Duke's criteria ${ }^{2}$ were applied to these case reports and papers were included if they met the criteria for definite or possible infective endocarditis caused by Pasteurella spp.

\section{Results of the Literature Search}

The search identified 28 papers and searching by citation identified a further six papers (Table 2). Of these, 32 papers met the authors' inclusion criteria (including case in this article), 31 described cases that met Duke's criteria for a definite diagnosis and one met the criteria for possible diagnosis of Pasteurella endocarditis." Of these there were 24 cases of $P$. multocida endocarditis, 3 -10,14-19,21-23,31-33 seven cases of non-multocida Pasteurella endocarditis, 9,21,26-28,30,31 and one case of undifferentiated Pasteurella endocarditis. ${ }^{7}$ Seven cases (21\%) had prosthetic valve endocarditis, 6,18-21,27 while 17 patients (53\%) had a predisposing cardiac condition 3 ,6,7,10,13,14,18,19,20,21,23,25,27 and 17 (53\%) had other underlying comorbidities reported. 3,5,6,10,14,-16,19,22,26,27,29-31

Twenty-one

patients (66\%) had documented animal exposure, $3,4,6,8,8,10,11,13,14,16-20,22,25-29$ of which 10 (31\%) reported a bite, scratch, or contact of saliva with broken skin.

Fever was the most common presenting symptom, afffecting 31 patients (97\%), and 18 patients $(56 \%)$ presented with systemic upset. Only 16 (50\%) had a cardiac murmur on initial presentation but 24 (75\%) developed complications, including 5 (16\%) with aortic root abscess and 13 (41\%) with septic emboli.

The diagnosis of infective endocarditis was missed in seven patients (22\%) and diagnosed on representation. Although the majority of patients presented within 7 days of symptom onset, nine patients (28\%) presented with a more prolonged history of general malaise, the longest being 3 months. ${ }^{10}$

Of the Pasteurella isolates, 19 patients (59\%) had susceptibility testing results reported and, of these, only one case reported penicillin resistance. $^{5}$ Antibiotic therapy included a $\beta$-lactam antibiotic in the majority of cases (24 patients, 75\%). Of the four patients who received penicillin or aminopenicillin monotherapy for the duration of treatment, three underwent valve surgery. Only one case had a reported allergy to penicillin and was treated successfully with 6 weeks of IV ceftriaxone. ${ }^{22}$

One patient with Pasteurella pneumotropica endocarditis of a native tricuspid valve was successfully treated with 6 weeks of ciprofloxacin monotherapy without surgery. ${ }^{27}$ Duration of treatment was reported in 25 cases (78\%). Of these cases, 20 patients (80\%) received at least 4 weeks of antibiotic therapy, with a median duration of 6 weeks (range: 1 day-20 weeks). Median duration of antibiotic therapy in patients who did not have surgery and survived was 5.8 weeks. Of the patients who did not survive, all apart from one were still on antimicrobial therapy when they died. ${ }^{5}$ 
Fourteen patients (44\%) underwent valve replacement surgery, and one was awaiting surgery. Indications for surgery included aortic root abscess in four patients (33\%), severe valvular regurgitation in seven (42\%), and persistent fever in three (17\%). Five out of seven (71\%) cases of prosthetic valve endocarditis were treated successfully, with three achieving cure on antibiotics alone, whilst the other two cases underwent surgical intervention.

Overall mortality rate from these cases was $19 \%$ (6/32). The mortality rate with surgery was $7 \%$ (1/14) and without surgery was $28 \%$ (5/18). Of the cases who died without surgery, two died within 24 hours of presentation and infective endocarditis was diagnosed at post-mortem examination; ${ }^{13,19}$ one had an aortic root abscess, a concurrent bacteraemia with Burkholderia cepacia and a candidaemia; 6 one received only 2 weeks of antibiotics and then died later from heart failure; 6 and one of the patients died 9 days prior to an operation being performed from severe heart failure. ${ }^{31}$ The patient who died after surgery presented with recurrence of infective endocarditis 6 weeks after his initial operation. The patient then represented after 4 months with a pseudomonal mitral and aortic valve endocarditis and died shortly after a further operation. ${ }^{20}$ Of the cases who did not have surgery, 14 (88\%) had underlying comorbidities compared with eight of the cases (57\%) who did have surgery.

\section{DISCUSSION}

A literature search found $P$. multocida to be a very rarely reported cause of infective endocarditis, with only 31 cases in the English language literature. The patient the authors' described was the first to be seen in their institution for over 20 years. This patient was also the first to suffer from septic arthritis as a complication. Although the authors' patient was successfully treated without surgery, they needed 8 weeks of antibiotics and the septic arthritis did not respond well to penicillin, requiring the addition of ciprofloxacin.

A recent analysis of Pasteurella spp. infective endocarditis cases concluded that all patients should be offered surgical intervention, unless an absolute contraindication exists given the difference in mortality rate. ${ }^{19}$ However, they did not include a patient who died 4 months post-valve replacement from early prosthetic valve endocarditis caused by Pseudomonas aeruginosa. ${ }^{20}$ The analysis was also limited by a lack of consideration of confounding factors such as comorbidity, which would skew the outcome in favour of surgery as surgical cases had a lower incidence of comorbidities. Furthermore, they did not adjust for severity of illness at presentation, nor whether existing guidelines were followed when considering surgical intervention. Therefore, the authors would suggest, as with other bacterial causes of infective endocarditis, that surgical intervention is not indicated in all cases, and should be decided on a case-by-case basis.

It is difficult to draw conclusions from the available literature as to the optimal choice and duration of antimicrobial therapy due to the significant variation in practice and the lack of reporting of antimicrobial susceptibility data or duration of antimicrobials. The authors' patient received an initial 24 days of penicillin monotherapy; however, due to persistent pyrexia and knee pain, the dose frequency of penicillin was increased, and oral ciprofloxacin was added. The patient improved clinically after this, but this could be attributed to a further joint washout. Although the patients required 8 weeks of therapy, this prolonged duration was likely required due to the infected joint. Based on the average duration of antibiotic therapy of 5.8 weeks in patients who survived without surgery, the authors would recommend 6 weeks of antibiotic therapy, depending on clinical response.

In summary, the authors have reported a patient who, despite significant comorbidities, including a pre-disposing cardiac condition, was successfully treated for $P$. multocida endocarditis and concurrent septic arthritis with an 8-week course of single and dual agent therapy of both penicillin and ciprofloxacin, without the need for cardiac surgery. It would be useful for future case reports to clearly document treatment duration as well as the indication for surgery or rationale for not performing surgery in order to build an evidence-base for treatment. The authors suggest that patients with Pasteurella spp. endocarditis can be managed according to 


\begin{tabular}{|c|c|c|c|c|c|c|c|c|c|c|c|c|}
\hline 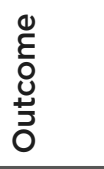 & $n$ & $\sim$ & 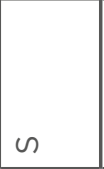 & ○ & 0 & $n$ & $\sim$ & $n$ & $n$ & $n$ & $\sim$ & $\sim$ \\
\hline 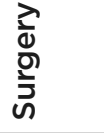 & $\stackrel{\mathscr{\omega}}{\nu}^{\mathscr{\omega}}$ & $\stackrel{\mathscr{\nu}}{\nu}^{\mathscr{N}}$ & $\stackrel{\mathscr{U}}{\nu}^{\mathscr{N}}$ & ㅇ & ㅇ & ㅇ & $\stackrel{\Perp}{\nu}^{\mathscr{U}}$ & $\stackrel{\varrho}{\nu}^{\mathscr{U}}$ & $\stackrel{\varrho}{\nu}$ & ㅇ & $\stackrel{\mathscr{\nu}}{\nu}^{\mathscr{\varrho}}$ & o \\
\hline 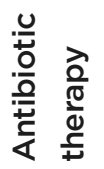 & 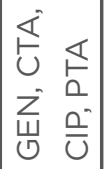 & $\begin{array}{l}z \\
\text { u } \\
z \\
\text { u }\end{array}$ & 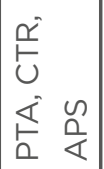 & 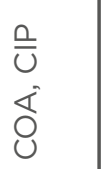 & 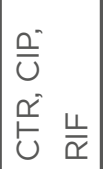 & 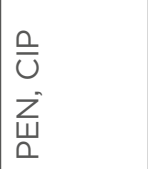 & 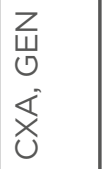 & $\sum_{<}^{O}$ & 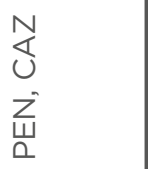 & $\begin{array}{l}\underline{x} \\
\text { U } \\
z ̇ \\
\underline{a}\end{array}$ & $\sum_{<}^{0}$ & zu \\
\hline 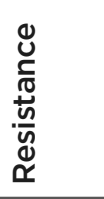 & $\overline{\bar{z}}$ & $\overline{\bar{z}}$ & $\underset{上}{\mathbb{b}}$ & そ & そ & $\overline{\bar{z}}$ & 吕 & 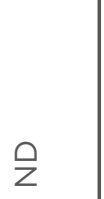 & $\overline{\bar{z}}$ & 吕 & $\overline{\bar{z}}$ & $\overline{\bar{z}}$ \\
\hline 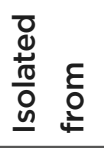 & U & u & U & U & U & 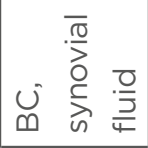 & u & $\underset{\infty}{\cup}$ & ৩ & $\underset{\infty}{u}$ & $\underset{\infty}{u}$ & $\underset{\infty}{\cup}$ \\
\hline $\begin{array}{l}\frac{\varepsilon}{\frac{1}{1}} \\
\frac{0}{0} \\
0 \\
0\end{array}$ & $\begin{array}{l}\frac{\pi}{2} \\
\frac{0}{0} \\
0 \\
\frac{1}{2} \\
\varepsilon \\
0 .\end{array}$ & $\begin{array}{l}\frac{0}{2} \\
\frac{1}{4} \\
0 \\
\frac{1}{2} \\
\varepsilon \\
0 . \\
0\end{array}$ & $\begin{array}{l}\frac{\pi}{2} \\
\frac{8}{0} \\
0 \\
\frac{2}{2} \\
\varepsilon \\
0 \\
\end{array}$ & $\begin{array}{l}\frac{\pi}{2} \\
\frac{8}{0} \\
0 \\
\frac{2}{2} \\
\frac{0}{2} \\
0\end{array}$ & $\begin{array}{l}\frac{\pi}{2} \\
\frac{0}{4} \\
0 \\
\frac{2}{2} \\
\varepsilon \\
0 \\
\end{array}$ & $\begin{array}{l}\frac{\pi}{2} \\
\frac{0}{4} \\
0 \\
\frac{1}{2} \\
\varepsilon \\
0 . \\
\end{array}$ & $\begin{array}{l}\frac{\pi}{2} \\
\frac{8}{4} \\
0 \\
\frac{1}{2} \\
\varepsilon \\
0 .\end{array}$ & 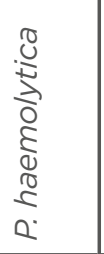 & $\begin{array}{l}\frac{\pi}{.0} \\
\frac{0}{0} \\
\frac{1}{5} \\
\varepsilon \\
0 .\end{array}$ & $\begin{array}{l}\frac{8}{2} \\
\frac{0}{4} \\
0 \\
\frac{1}{5} \\
\varepsilon \\
0 .\end{array}$ & $\begin{array}{l}\frac{\pi}{20} \\
\frac{0}{0} \\
\frac{1}{5} \\
\varepsilon \\
0 .\end{array}$ & 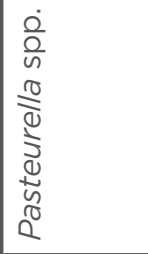 \\
\hline $\begin{array}{l}0 \\
\stackrel{0}{\bar{y}} \\
0 \\
\dot{0} \\
\dot{x} \\
\text { x }\end{array}$ & 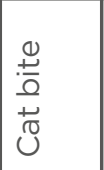 & 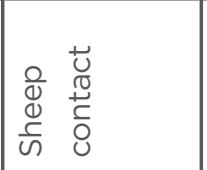 & $\overline{\bar{z}}$ & $\begin{array}{l}\frac{0}{0} \\
\frac{0}{0} \\
\frac{1}{0} \\
ن\end{array}$ & $\overline{\bar{z}}$ & 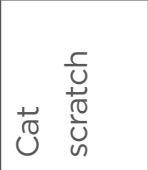 & 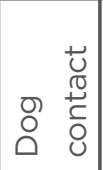 & $\begin{array}{l}8 \\
0 \\
0 \\
+0 \\
0\end{array}$ & $\begin{array}{l}8 \\
\frac{0}{0} \\
+0 \\
0\end{array}$ & $\overline{\bar{z}}$ & o & 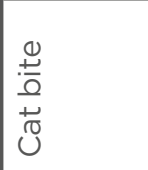 \\
\hline 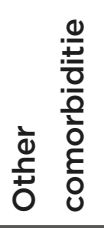 & $\stackrel{\Perp}{\circlearrowright}$ & $\stackrel{\circ}{z}$ & $\stackrel{\varrho}{\nu}$ & $\stackrel{\varrho}{\rightleftharpoons}$ & $\stackrel{\varrho}{\rightleftharpoons}$ & $\stackrel{\varrho}{\rightleftharpoons}$ & $\stackrel{\mathscr{\Perp}}{\rightleftharpoons}$ & zo & ㅇ & 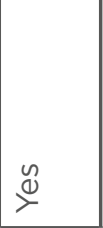 & $\stackrel{\bigotimes}{\circlearrowright}$ & zo \\
\hline 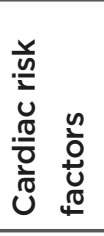 & 尔 & $\overline{\bar{z}}$ & $\overline{\bar{z}}$ & $\overline{\bar{z}}$ & 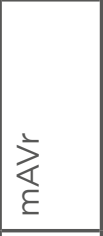 & $\begin{array}{l}\sum^{n} \\
\sum_{\Sigma} \\
\varepsilon\end{array}$ & $\overline{\bar{z}}$ & $\overline{\bar{z}}$ & $\Sigma^{N}$ & $\overline{\bar{z}}$ & 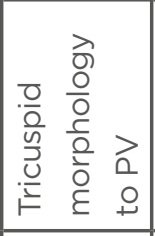 & 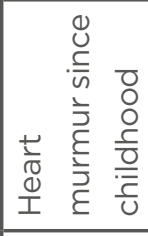 \\
\hline 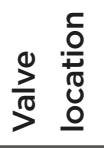 & $\varangle$ & $\varangle$ & $\Sigma$ & $\Sigma$ & $\varangle$ & $\Sigma$ & $\varangle$ & $\varangle$ & $\Sigma$ & $\varangle$ & $\bar{\alpha}$ & c. \\
\hline 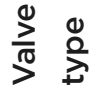 & $z$ & $z$ & $z$ & $z$ & $\bar{\alpha}$ & $z$ & $z$ & $z$ & $z$ & $z$ & $z$ & $z$ \\
\hline 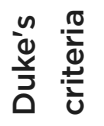 & 0 & o & 0 & 0 & 0 & 0 & o & ○ & 0 & o & 0 & 0 \\
\hline 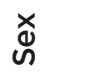 & $\Sigma$ & $\Sigma$ & $\Sigma$ & $\sqcup$ & $\sqcup$ & $\sqcup$ & $\Sigma$ & $\Sigma$ & $\Sigma$ & ч & レ & $\Sigma$ \\
\hline 导 & $\stackrel{\circ}{\wedge}$ & 으 & 윰 & $\stackrel{\curvearrowright}{\wedge}$ & $\bar{\infty}$ & $\stackrel{\Omega}{\wedge}$ & $\stackrel{\infty}{+}$ & $\widetilde{\sigma}$ & $\stackrel{\infty}{+}$ & $\stackrel{\infty}{M}$ & $\stackrel{\varphi}{M}$ & $\stackrel{\infty}{+}$ \\
\hline 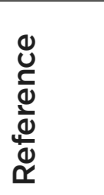 & 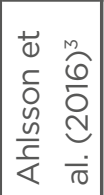 & 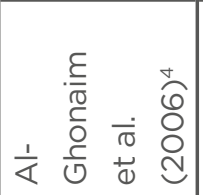 & 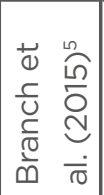 & 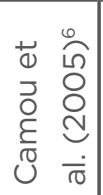 & 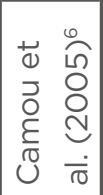 & 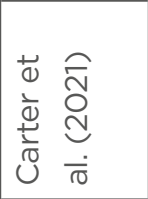 & 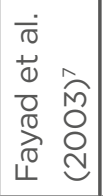 & 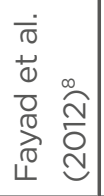 & 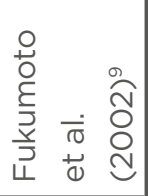 & 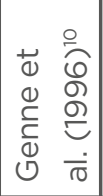 & 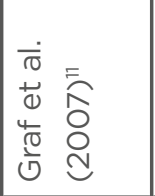 & 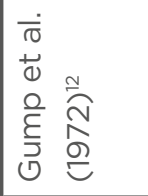 \\
\hline
\end{tabular}




\begin{tabular}{|c|c|c|c|c|c|c|c|c|c|c|c|}
\hline 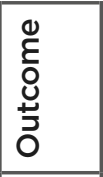 & 0 & 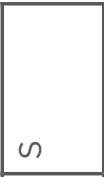 & $n$ & $n$ & $\sim$ & $\sim$ & $n$ & 0 & $n$ & $n$ & $n$ \\
\hline $\begin{array}{l}\frac{\pi}{0} \\
0 \\
0 \\
\vdots \\
\omega\end{array}$ & ㅇ & ㅇ & $\stackrel{\tilde{\nu}}{\nu}$ & $\stackrel{\tilde{u}}{>}$ & ㅇ & $\stackrel{\circ}{z}$ & $\stackrel{\tilde{e}}{\nu}$ & $\stackrel{\tilde{y}}{\nu}$ & ㅇ & $\stackrel{\tilde{\nu}}{\succ}$ & $\stackrel{\circ}{z}$ \\
\hline 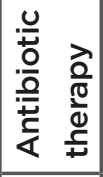 & 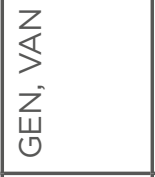 & $\begin{array}{l}Z \\
\text { U } \\
0 \\
\sum_{<} \\
\end{array}$ & 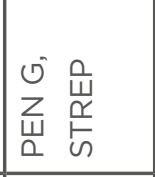 & $\begin{array}{l}0 \\
\frac{0}{4}\end{array}$ & $\begin{array}{l}\frac{a}{U} \\
Z \\
\underline{a} \\
\end{array}$ & $\begin{array}{l}n \\
\frac{n}{4} \\
z \\
\ddot{\alpha} \\
\vdots\end{array}$ & 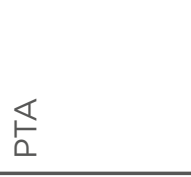 & $\sum_{<}^{0}$ & $\frac{r}{v}$ & 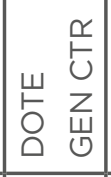 & $\stackrel{\alpha}{\underline{u}}$ \\
\hline 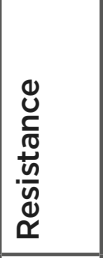 & 吕 & Z & 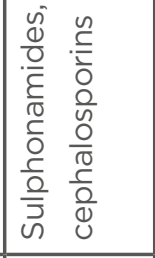 & $\overline{\bar{z}}$ & $\overline{\bar{z}}$ & $\overline{\bar{z}}$ & 乏 & $\overline{\bar{z}}$ & 乏 & $\overline{\bar{z}}$ & 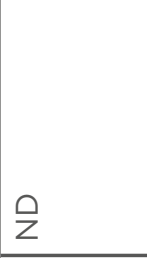 \\
\hline 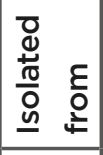 & u & U. & U. & U. & $\begin{array}{|ll|}0 & \\
\frac{0}{\sigma} & \\
0 & n \\
\infty & 0 \\
\end{array}$ & $\cup$ & U & $\bigcup_{\infty}$ & U & $\underset{\infty}{u}$ & $\bigcup_{\infty}$ \\
\hline 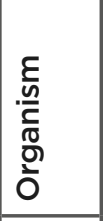 & 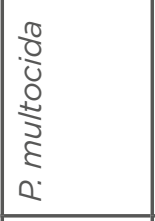 & 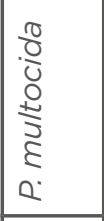 & 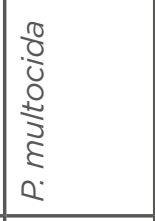 & 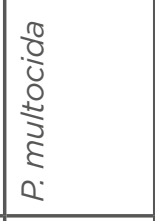 & \begin{tabular}{|l}
0 \\
$\frac{\pi}{0}$ \\
0 \\
0 \\
$\frac{1}{3}$ \\
8 \\
0 \\
0
\end{tabular} & $\begin{array}{l}0 \\
\frac{0}{0} \\
0 \\
0 \\
\frac{1}{3} \\
0 \\
0 \\
\end{array}$ & 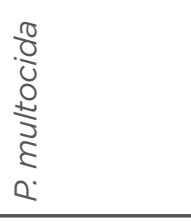 & 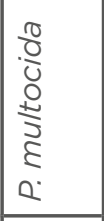 & 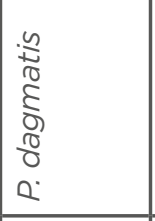 & 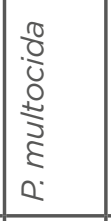 & $\begin{array}{l}\frac{\pi}{0} \\
\frac{1}{4} \\
0 \\
\frac{2}{2} \\
\varepsilon \\
0 \\
\end{array}$ \\
\hline 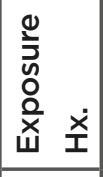 & 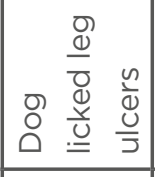 & 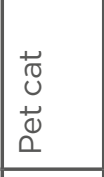 & $\overline{\bar{z}}$ & 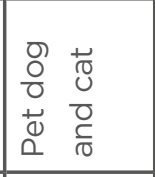 & 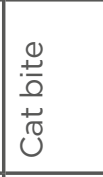 & $\begin{array}{l}\overrightarrow{0} \\
0 \\
\overrightarrow{0} \\
0 \\
\end{array}$ & $\begin{array}{l}+\overrightarrow{0} \\
0 \\
+0 \\
0\end{array}$ & 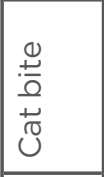 & $\overline{\bar{z}}$ & 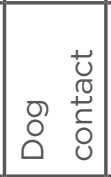 & $\overline{\bar{z}}$ \\
\hline 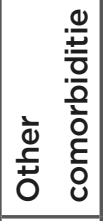 & 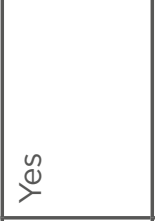 & $\stackrel{\Perp}{\nu}$ & ํ. & ㅇ & $\stackrel{\tilde{\omega}}{\nu}$ & z & $\stackrel{\tilde{N}}{\nu}$ & z & ¿̊ & z & $\stackrel{\Perp}{\nu}$ \\
\hline 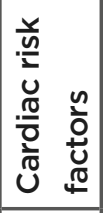 & 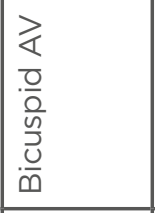 & 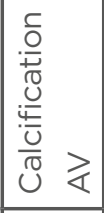 & $\overline{\bar{z}}$ & $\overline{\bar{z}}$ & $\overline{\bar{z}}$ & $\stackrel{1}{\$}$ & 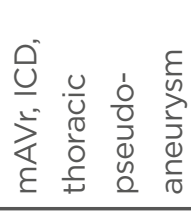 & 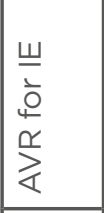 & $\stackrel{2}{\$}$ & $\overline{\bar{z}}$ & 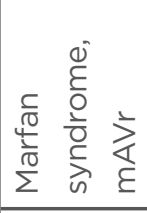 \\
\hline 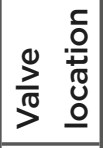 & $\varangle$ & $\varangle$ & $\varangle$ & $\varangle$ & $\vdash$ & $\varangle$ & $\varangle$ & $\varangle$ & $\varangle$ & $\varangle$ & $\underset{\nwarrow}{\nwarrow}$ \\
\hline 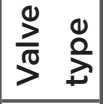 & $z$ & $z$ & $z$ & $z$ & $z$ & $\grave{\Delta}$ & $\bar{\alpha}$ & $\grave{\Delta}$ & $\grave{\Delta}$ & $z$ & n. \\
\hline 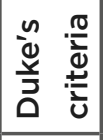 & O & 0 & 0 & 0 & 0 & 0 & 0 & 0 & 0 & 0 & 0 \\
\hline 爻 & $\Sigma$ & $\Sigma$ & $\Sigma$ & \llcorner & $\sqcup$ & $\sqcup$ & 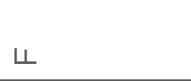 & $\Sigma$ & $\Sigma$ & $\sqcup$ & $\Sigma$ \\
\hline 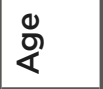 & $\overline{6}$ & $\underset{\infty}{\infty}$ & n & 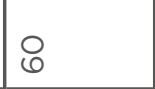 & $\begin{array}{l}\infty \\
\infty\end{array}$ & $\aleph$ & $\mathscr{\bullet}$ & $\mathscr{\bullet}$ & $\stackrel{\infty}{\wedge}$ & $\hat{\theta}$ & $\begin{array}{l}\infty \\
M\end{array}$ \\
\hline 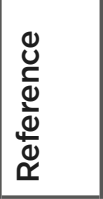 & 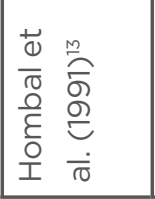 & 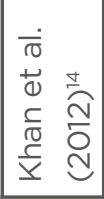 & 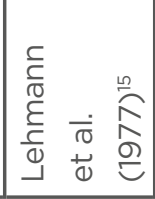 & 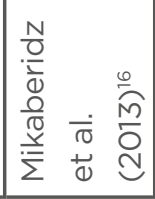 & 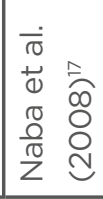 & 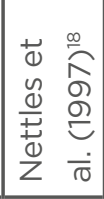 & 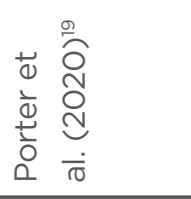 & 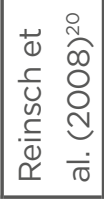 & 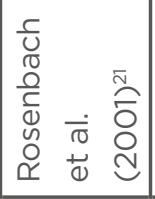 & 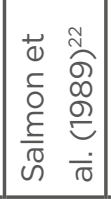 & 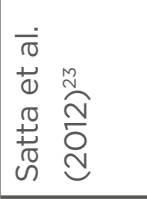 \\
\hline
\end{tabular}




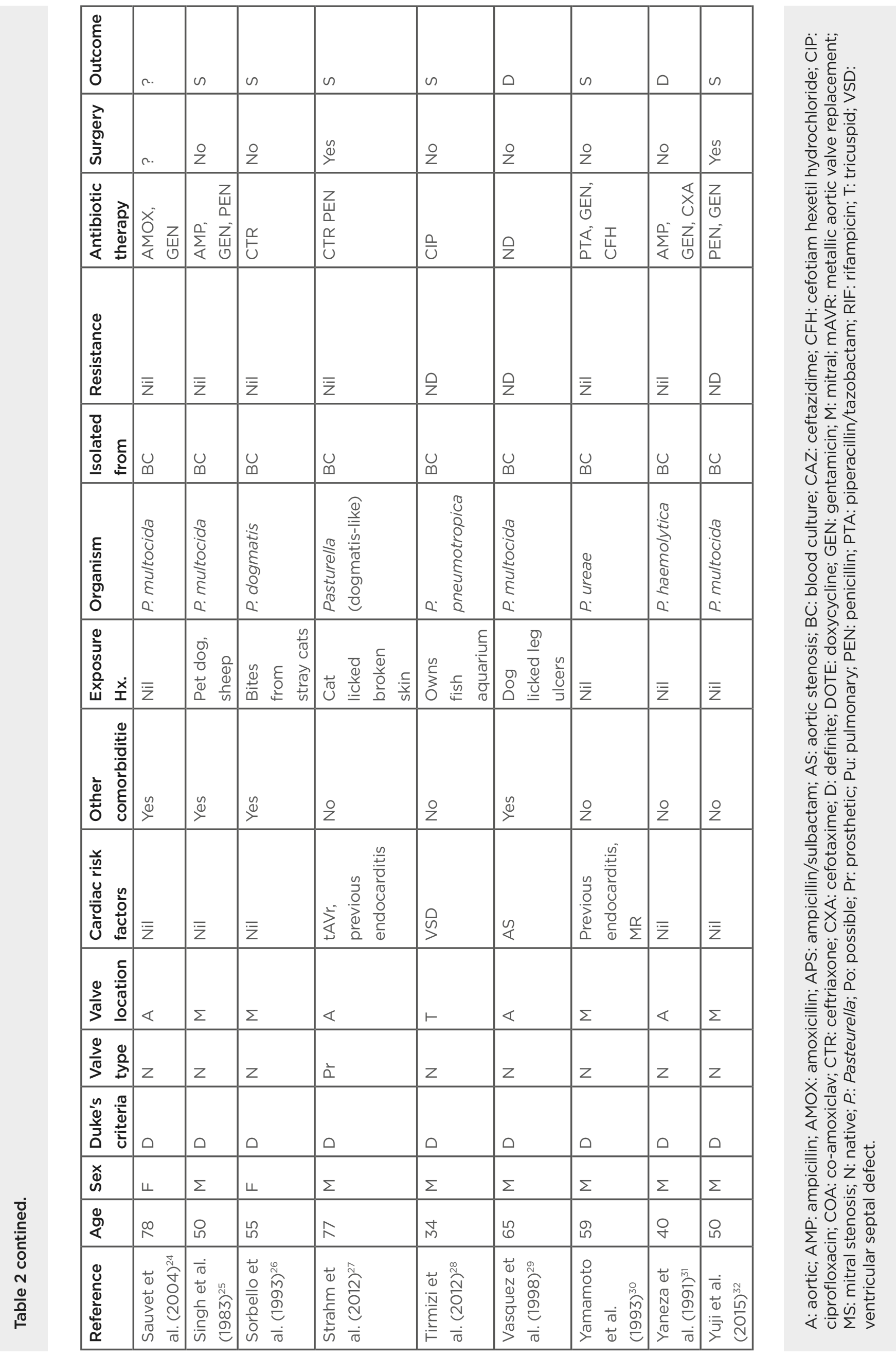


available infective endocarditis guidelines, which include the involvement of a dedicated infective endocarditis team, who assess the need for surgical intervention on a case-by-case basis. This adds to the small number of case reports in the literature that describe successful management of Pasteurella spp. endocarditis and may help inform other clinicians when they encounter similar cases.

\section{References}

1. Bennett JE et al. Mandell, Douglas, and Bennett's Principles and practice of infectious diseases. 2015. $9^{\text {th }}$ edition, Philadelphia: Elsevier/Saunders.

2. Li JS et al. Proposed modifications to the Duke criteria for the diagnosis of infective endocarditis. Clin Infect Dis. 2000;30(4):633-8.

3. Ahlsson A et al. An angry cat causing Pasteurella multocida endocarditis and aortic valve replacement-a case report. Int J Surg Case Rep. 2016;24:91-3.

4. Al-Ghonaim MA et al. Endocarditis caused by Pasteurella multocida. Ann Saudi Med. 2006;26(2):147-9.

5. Branch J et al. Pasteurella multocida infective endocarditis: a possible link with primary upper respiratory tract infection. Internal Med. 2015:54(24):3225-31.

6. Camou F et al. [Endocarditis due to Pasteurella sp. two cases]. Med Mal Infect. 2005;35(11):556-9. (In French)

7. Fayad G et al. Pasteurella multocida aortic valve endocarditis: case report and literature review. J Heart Valve Dis. 2003;12(2):261-3.

8. Fayad $\mathrm{G}$ et al. First documented surgical case of human aortic valve endocarditis caused by Pasteurella haemolytica. J Heart Valve Dis. 2012;21(1):139.

9. Fukumoto $Y$ et al. Pasteurella multocida endocarditis: report of a case. Surg Today. 2002;32(6):513-5.

10. Genne D et al. Pasteurella multocida endocarditis: report of a case and review of the literature. Scand J Infect Dis. 1996;28(1):95-7.

11. Graf $\mathrm{S}$ et al. Isolated endocarditis of the pulmonary valve caused by Pasteurella multocida. Infection. 2007;35(1):43-5.
12. Gump DW, Holden, RA. Endocarditis caused by a new species of Pasteurella. Ann Intern Med. 1972;76(2):275-8.

13. Hombal SM, Dincsoy HP. Pasteurella multocida endocarditis. Am J Clin Pathol. 1992;98(6):565-8.

14. Khan MF et al. Pasteurella multocida endocarditis. J Heart Valve Dis. 2012;21(2):260-2.

15. Lehmann $\vee$ et al. Endocarditis caused by Pasteurella multocida. Scand J Infect Dis. 2015;9(3):247-8.

16. Mikaberidz $\mathrm{N}$ et al. Pasteurella multocida infective endocarditis in an immunocompetent patient complicated by rhabdomyolysis and permanent hearing loss. J Cardiovasc Dis Res. 2013;4(1):55-7.

17. Naba MR et al. First case of Pasteurella multocida endocarditis of the tricuspid valve: a favorable outcome following medical treatment. International journal of infectious diseases. Int $\mathrm{J}$ Infect Dis. 2009;13(5):267-9.

18. Nettles RE, Sexton DJ. Pasteurella multocida prosthetic valve endocarditis: case report and review. Clin Infect Dis. 1997;25(4):920-1.

19. Porter RS, Hay CM. Pasteurella endocarditis: a case report and statistical analysis of the literature. Case Rep Infect Dis. 2020;2020:8890211.

20. Reinsch $\mathrm{N}$ et al. Recurrent infective endocarditis with uncommon gramnegative Pasteurella multocida and Pseudomonas aeruginosa: a case report. J Heart Valve Dis. 2008;17(6):710-3.

21. Rosenbach KA et al. Prosthetic valve endocarditis caused by Pasteurella dagmatis. South Med J. 2001;94(10):1033-5.

22. Salmon D et al. Endocarditis due to pasteurella multocida with glomerulonephritis. Am J Med. 1989;86(4):493.

23. Satta $\mathrm{G}$ et al. Prosthetic valve endocarditis caused by Pasteurella in a penicillin allergic patient: challenges in diagnosis and treatment. Infect Dis Rep. 2012;4(1):32.

24. Sauvet F et al. [Pasteurella multocida endocarditis revealed by inflammatory rachialgia]. Rev Med Interne. 2004;25(7):530-1. (In French).

25. Singh CP, Spurrell JR. Pasteurella multocida endocarditis. Br Med J (Clin Res Ed). 1983;286(6381):1862-3.

26. Sorbello AF et al. Infective endocarditis due to Pasteurella dagmatis: case report and review. Clin Infect Dis. 1994;18(3):336-8.

27. Strahm $\mathrm{C}$ et al. Prosthetic valve endocarditis caused by a Pasteurella dagmatis-like isolate originating from a patient's cat. J Clin Microbiol. 2012;50(8):2812-9.

28. Tirmizi A et al. First reported case of Pasteurella pneumotropica tricuspid valve endocarditis. Int J Cardiol. 2012;161(3):e44-5.

29. Vasquez JE et al. Pasteurella multocida endocarditis: a molecular epidemiological study. Clin Infect Dis. 1998;26(2):518-20.

30. Yamamoto $\mathrm{K}$ et al. Pasteurella ureae endocarditis. Intern Med. 1993;32(11):872-4.

31. Yaneza AL et al. Pasteurella haemolytica endocarditis. J Infect. 1991;23(1):65-7.

32. Yuji D et al. Pasteurella multocida infective endocarditis. J Heart Valve Dis. 2015;24(6):778-9.

33. Guerain JM et al. [Pasteurella multocida endocarditis]. Nouv Presse Med. 1980;9(37):2735-6. (In French). 